\title{
Blind Ratio Combining (BRC): An Optimum Diversity Receiver for Coherent Detection With Unknown Fading Amplitudes
}

\author{
Athanasios S. Lioumpas, Student Member, IEEE, and George K. Karagiannidis, Senior Member, IEEE
}

\begin{abstract}
We present an optimum diversity receiver called blind ratio combining (BRC) that minimizes the average symbol error probability or maximizes the average output SNR, where the channels' time delays and the random phases are known, while the fading amplitudes are unknown. In contrast to previous works, where efforts were made to find a posteriori probabilities at the receiver, the BRC simply calculates the optimum weights, which depend on the channel's statistics, avoiding continuous channel estimation, and thus, it significantly reduces the system's complexity. In nonidentical multipath fading channels with power delay profile (PDP), the BRC receiver performs between maximal ratio combining (MRC) and equal gain combining (EGC), and keeps its performance comparable-and in some cases superior- to that of generalized selection combining, while for large values of the decay factor, it approaches MRC. Moreover, in the important practical case of exponential PDP-common in RAKE receivers modeling and adopted for the Universal Mobile Telecommunications System spatial channel modeling by the European Telecommunications Standards Institute-3GPP - the optimum weights can be accurately approximated by simple elementary functions. Furthermore, it is proved that the utilization of these weights ensures an error performance improvement over EGC for arbitrary PDPs. The proposed $B R C$ receiver can be efficiently applied in wireless wideband communication systems, where a large number of diversity branches exists, due to the strong multipath effects.
\end{abstract}

Index Terms-Blind ratio combining (BRC), diversity techniques, equal gain combining (EGC), fading channels, generalized selection combining (GSC), maximal ratio combining (MRC).

\section{INTRODUCTION}

$\mathbf{M}$ ULTICHANNEL receivers followed by certain diversity combining techniques can significantly improve the system's performance and are of great interest, even more in the emerging broadband communication systems (e.g., ultrawideband), where the number of diversity paths can be considerably large due to the strong multipath effects. The diversity combining basically relies on the reception of the replicas of

Paper approved by V. A. Aalo, the Editor for Transmission Systems of the IEEE Communications Society. Manuscript received May 10, 2006; revised October 16, 2006 and January 8, 2007. This work was supported in part by the General Secretariat for Research and Technology (GSRT) of the Hellenic Ministry of Development under a grant for the Bilateral S\&T Cooperation between the Hellenic Republic and Montenegro for the years 2005-2007. It was also performed within the framework of the Satellite Communications Network of Excellence (SatNEx) project (IST-507052) and its Phase-II, SatNEx-II (IST27393), funded by the European Commission (EC) under its FP6 program.

The authors are with the Division of Telecommunications, Department of Electrical and Computer Engineering, Aristotle University of Thessaloniki, 54124 Thessaloniki, Greece (e-mail: alioumpa@auth.gr; geokarag@auth.gr).

Digital Object Identifier 10.1109/TCOMM.2007.904378 the same information distorted by independent (or near independent) fading paths.

Among the well-known diversity techniques, maximal ratio combining (MRC) [1]-[3] is the optimal combiner in the sense that it attains the highest output signal-to-noise ratio (SNR) of any combining scheme, independent of the distribution of the branch signals [4]. However, it requires the knowledge of the channel (path) fading amplitudes and phases of each diversity branch, which in practice are not known and must be continuously estimated by the receiver, resulting in high complexity. Alternative combining techniques such as equal gain combining (EGC) [5]-[12], selection combining (SC) [13], [14], or switch combining (SWC) [15]-[18] are often used in practice due to their reduced complexity relative to the optimum MRC scheme. Indeed, EGC weights each branch equally before combining, and therefore, does not require the estimation of the channel fading amplitudes, while its error performance is comparable to that of the MRC for certain fading environments [not severe independent identically distributed (i.i.d.) fading channels]. Otherwise, and especially in environments with power delay profile (PDP), the performance of the EGC can dramatically deteriorate, due to the combining loss: combining more signals does not necessarily enhance performance, especially for high bit error rates (BERs), since the weak branches contribute more in increasing the noise than the signal power [19]. In such fading environments, the generalized selection combining (GSC) constitutes as an alternative solution with better performance than EGC and lower complexity compared to MRC. A comprehensive summary of the diversity combining methods can be found in [4].

Particular interest has been given in the past in the design and specification of optimum (a posteriori probability) receivers, which optimize the system's performance with respect to some a priori chosen performance criterion [20]-[23]. In these pioneering works, the proposed optimum receivers compute the set of a posteriori probabilities, which depend on the degree of knowledge of the channels' parameters, i.e., the fading amplitude, the random phase, and the time delay, introduced by the fading medium. However, in many instances, implementation of the optimum structure may not be simple or even feasible [4, p. 189]. Special efforts were made for the case where the receiver has full knowledge of both the time delays and phases but not of the channel gains, since the measurement of the phase characteristic is more important than measuring the channel gain, concerning the error performance [20]. Most of these works dealt with only binary modulation/detection schemes 
(i.e., BPSK with coherent detection in Rayleigh or Rician fading channels). Moreover, practical receiver's structure was given only for BPSK modulation in Rayleigh and Nakagami- $m$ fading for the high SNR region, since the calculation of the a posteriori probabilities is not always an easy task.

In this paper, we present a simpler structure for an optimum diversity receiver with coherent detection, called blind ratio combining (BRC), which minimizes the average symbol error probability (ASEP), or maximizes the average output SNR, when the fading amplitudes are not available at the receiver. In contrast to previous works, where efforts were made to find a posteriori probabilities, the new proposed receiver simply calculates the optimum weights at each diversity branch, contributing in minimizing the combining loss, and consequently, minimizing the ASEP and maximizing the combined average SNR (ASNR). Its structure is similar to that of the MRC receiver, but the weights do not depend on the instantaneous channel gains (thus avoiding continuous channel estimation) but on the long-term channel statistics. This can be efficiently estimated at the initialization stage, before the communication begins and remains either almost constant in general or changes slowly in such a manner that it can be accurately tracked at the receiver [24]. Observing that BRC constitutes a virtual EGC receiver, previously published formulas for the error performance of the EGC receivers [6]-[8] can be efficiently applied to evaluate the optimum weights, solving a minimization problem by means of well-known numerical methods.

Considering multipath fading channels with PDP, the optimum weights can be accurately approximated by simple elementary functions, and thus, the complexity of the $\mathrm{BRC}$ receiver can be significantly reduced - avoiding to solve the minimization problem. Moreover, it is proved by using the majorization theory [25] that the utilization of these weights ensures an error performance improvement over EGC for arbitrary PDPs. The $\mathrm{BRC}$ performs between MRC and EGC and keeps its performance comparable to that of the GSC, while for large values of the decay factor, it approaches MRC. This happens in contrast to EGC, whose error performance dramatically decreases with an increase of the number of branches, when operating over nonidentical fading channels.

The BRC receiver proposed in this paper can be efficiently applied in wideband communication systems, where a large number of diversity branches exist, due to the strong multipath effects. In these systems, the propagation environment varies widely from strong line-of-sight to extreme non-line-of sight, where the multipaths may be nonidentically distributed, concerning not only their average powers, but also their fading parameters [26], [27].

The outline of the paper is as follows. Section II describes the BRC system model, while the computation of the optimum weights is presented in Section III for the Rayleigh and Nakagami- $m$ fading models employing $M$-PSK modulation. In Section IV, considering exponential PDP, the optimum weights are accurately approximated by elementary functions, while Section $\mathrm{V}$ provides a comparison of the BRC with the MRC, EGC, and GSC receivers. Finally, concluding remarks are presented in Section VI.

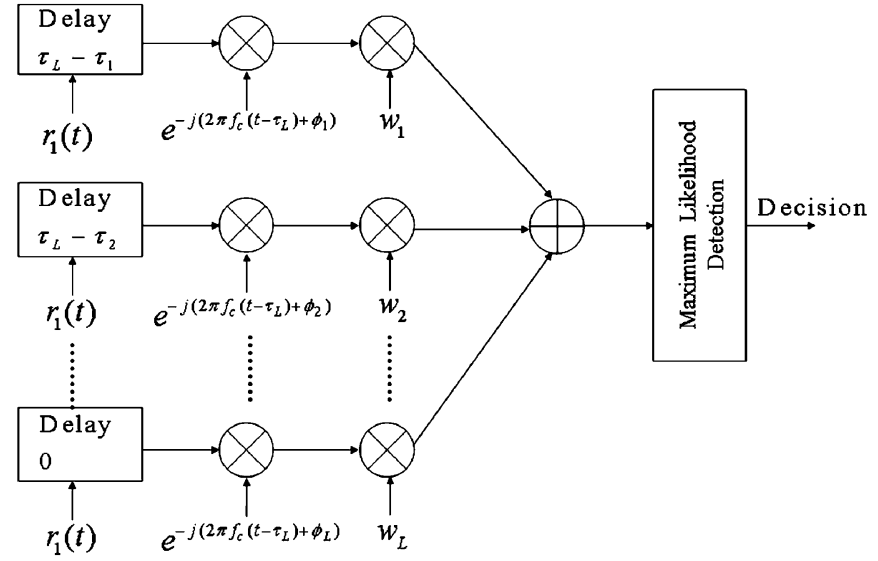

Fig. 1. A coherent multichannel receiver. MRC: $w_{k}=a_{k} / N_{0}$. EGC: $w_{k}=$ 1. BRC: $w_{k}$ depends on the long-term channel's statistics.

\section{SySTEM MODEL}

Consider a multichannel diversity reception system with $L$ branches operating in a flat fading environment, in which the receiver employs symbol-by-symbol detection. The signal received over the $k$ th diversity branch in a symbol interval of duration $T_{S}$ can be expressed as

$$
\begin{aligned}
r_{k}(t) & =\Re\left\{\left[a_{k}\left(t-\tau_{k}\right) e^{-j \varphi_{k}(t)} s(t)+n_{k}(t)\right] e^{j 2 \pi f_{c} t}\right\} \\
& =\Re\left\{R_{k}(t) e^{j 2 \pi f_{c} t}\right\}, \quad k=1, \ldots, L
\end{aligned}
$$

where $s(t)$ is the complex baseband information-bearing signal with average symbol energy $2 E_{s}, a_{k}(t)$ is the random magnitude, $\varphi_{k}(t)$ and $\tau_{k}$ are the random phase and delay of the $k$ th diversity branch, $f_{c}$ is the carrier frequency and $n_{k}(t)$, representing the additive noise, is a zero-mean complex Gaussian random process with two-sided power spectral density $2 N_{0}$.

Assuming that the random phase $\varphi_{k}(t)$ and the delay $\tau_{k}$ are known at the receiver, the received signals are cophased and transferred to baseband so that the signal at the $k$ th branch will be

$$
u_{k}(t)=\Re\left\{a_{k}(t) s(t)+n_{k}(t) e^{j \varphi_{k}(t)}\right\}, \quad k=1, \ldots, L .
$$

At the combination stage, the signals $u_{k}(t)$ are weighted and summed to produce the decision variable

$$
u(t)=\sum_{k=1}^{L} w_{k}(t) u_{k}(t)
$$

where $w_{k}(t)$ is the weight of the $k$ th branch. Then, by applying maximum likelihood detection (MLD), the combiner's output is compared with all the known possible transmitted symbols in order to extract the decision metric. The general structure of the previously presented multichannel receiver is depicted in Fig. 1. For the reader's convenience, the time notation will be omitted in the following.

\section{A. MRC and EGC}

When MRC is used, the received signal at the $k$ th branch is weighted by the instantaneous channel gain at that time interval, i.e., $w_{k}=a_{k} / N_{k}$, where $N_{k}$ is the mean square noise power of 
the $k$ th branch. These optimum weights are obtained by applying continuous channel estimation at the receiver, and as a result, the output SNR is given by [4]

$$
\gamma_{\mathrm{MRC}}=\sum_{k=1}^{L} \gamma_{k}
$$

where $\gamma_{k}$ is the instantaneous SNR of the $k$ th branch.

On the contrary, coherent EGC cophases and equally weights each branch before combining, and therefore, does not require estimation of the channel (path) fading amplitudes, but only the knowledge of the channel phase, in order for the demodulator to undo the random phase shifts introduced on the diversity channels. As a result, the output SNR is given by [4]

$$
\gamma_{\mathrm{EGC}}=\frac{\left(\sum_{k=1}^{L} a_{k}\right)^{2} E_{s}}{\sum_{k=1}^{L} N_{k}}
$$

and if it is assumed that $N_{k}=N_{0}$, (5) is simplified to

$$
\gamma_{\mathrm{EGC}}=\frac{\left(\sum_{k=1}^{L} \sqrt{\gamma_{k}}\right)^{2}}{L} .
$$

\section{B. $B R C$}

In $\mathrm{BRC}$ receivers proposed in this paper, it is assumed that the received signal at the $k$ th branch is weighted by the constant quantity $w_{k}$ over a certain time period. Following the same procedure as in MRC or EGC, the output SNR can be finally expressed as

$$
\gamma_{\mathrm{BRC}}=\frac{\left(\sum_{k=1}^{L} w_{k} a_{k}\right)^{2} E_{s}}{\sum_{k=1}^{L} w_{k}^{2} N_{k}}
$$

and if $N_{k}=N_{0}$, the output SNR of the BRC is given by

$$
\gamma_{\mathrm{BRC}}=\frac{\left(\sum_{k=1}^{L} w_{k} \sqrt{\gamma_{k}}\right)^{2}}{\sum_{k=1}^{L} w_{k}^{2}} .
$$

The weights $w_{k}$ depend on the channel's statistics (thus avoiding continuous channel estimation) and can be calculated at the initialization stage before the communication begins, remaining constant as long as the channel statistics do not change. It must be noted here that the rate at which channel statistics change depends on the large-scale variations in the scattering environment and is typically constant or changes slowly, so it can be accurately tracked at the receiver and can be estimated reliably [24]. From (8) and after some manipulations, the BRC output SNR can be written as

$$
\gamma_{\text {out }}=\frac{\left(\sum_{k=1}^{L} \sqrt{b_{k}^{2} \gamma_{k}}\right)^{2}}{L}
$$

where

$$
b_{k}=w_{k} \sqrt{\frac{L}{\sum_{k=1}^{L} w_{k}^{2}}} .
$$

Comparing (6) and (9), it can be easily observed that the BRC receiver constitutes a virtual $E G C$ receiver, where the random variable $(R V) \gamma_{k}$ is replaced by the $R V b_{k}^{2} \gamma_{k}$.

Also note that (7) describes the instantaneous output SNR of a general $L$-branch diversity combining receiver, which reduces to the MRC receiver for $w_{k}=a_{k} / N_{k}$, or to the EGC receiver for $w_{k}=a$, where $a$ is a constant.

\section{OPtIMAL WeIGHTS FOR BRC RECEIVERS}

Let the channel (path) fading amplitudes $\left\{a_{k}\right\}_{k=1}^{L}$ at each of the $L$ diversity branches of the BRC receiver be represented by the vector $\mathbf{a}=\left[\begin{array}{llll}a_{1} & a_{2} & \cdots & a_{k}\end{array}\right]$ and the weights $\left\{w_{k}\right\}_{k=1}^{L}$ applied to each diversity branch by the vector $\mathbf{w}=$ $\left[\begin{array}{llll}w_{1} & w_{2} & \cdots & w_{k}\end{array}\right]$. The problem we set here is to find the optimum weights $w_{k}$ per branch that depend only on the channel's statistics (and not on the instantaneous channel gains) and minimize the ASEP or maximize the combined ASNR.

\section{A. Minimization of the ASEP}

Since the BRC receiver constitutes a virtual EGC receiver, the symbol error probability conditioned on the fading amplitudes can be written in the form

$$
P_{e}\left(E \mid\left\{a_{k}\right\}_{k=1}^{L}\right)=\mathcal{G}(\mathbf{w}, \mathbf{a})
$$

where $\mathcal{G}(\cdot)$ denotes a function that depends on the coherent modulation scheme (e.g., $M$-PSK) and the fading channel model. For example, when BPSK modulation is used, $\mathcal{G}(\mathbf{w}, \mathbf{a})$ is given by $[4$, p. 189]

$$
\mathcal{G}(\mathbf{w}, \mathbf{a})=Q\left(\sqrt{\frac{2 E_{s}}{\sum_{k=1}^{L} w_{k}^{2} N_{0}}\left(\sum_{k=1}^{L} w_{k} a_{k}\right)^{2}}\right)
$$

where $Q(\cdot)$ is the Gaussian $Q$-function and $E_{s}$ is the average energy per symbol. The ASEP $P_{e}(E)$ is obtained by averaging (11) over the joint probability density function (pdf) of the channel fading amplitudes $p_{\mathbf{a}}(\mathbf{a})$, i.e.,

$$
P_{e}=\int_{0}^{\infty} \mathcal{G}(\mathbf{w}, \mathbf{a}) p_{\mathbf{a}}(\mathbf{a}) d \mathbf{a} .
$$

The problem described before is to find the vector $\Omega=$ $\left[\begin{array}{llll}\Omega_{1} & \Omega_{2} & \cdots & \Omega_{k}\end{array}\right]$, which minimizes (13), i.e.,

$$
\begin{gathered}
\boldsymbol{\Omega}=\underset{\mathbf{w}}{\arg \min }\left\{P_{e}\right\} \\
\text { s.t. } w_{k} \in\left(l_{1}, l_{2}\right), l_{1}, l_{2} \in R^{+} .
\end{gathered}
$$

The constraint in (14) is necessary due to the fact that any vector proportional to $\Omega$ also constitutes a solution to the minimization problem, since the vector $a \boldsymbol{\Omega}$, with $a$ a constant number, would result in the same output SNR given by (7). We have to mention here that the weights should belong to the subspace $(0,1)$ in the sense that the received signals are not amplified, in order to avoid extra power consumption.

1) BPSK and Rayleigh Fading: The extraction of the weights $\Omega_{k}$ by directly minimizing (13) is not an easy task, since the pdf $p_{\mathbf{a}}(\mathbf{a})$ is yet unknown in a closed form. Fortunately, for BPSK modulation in Rayleigh fading, Zhang [6] presented an analytical formula for the ASEP as

$$
P_{e}=\frac{1}{2}-\frac{1}{\pi} \int_{0}^{\infty} H(z) \exp \left(-z^{2}\right) d z
$$


with

$$
\begin{aligned}
H(z) & =\operatorname{Im}\left\{\prod _ { k = 1 } ^ { L } \left[{ }_{1} F_{1}\left(-\frac{1}{2} ; \frac{1}{2} ; \frac{b_{k}^{2} \bar{\gamma}_{k} z^{2}}{L+\sum_{i=1}^{L} b_{i}^{2} \bar{\gamma}_{i}}\right)\right.\right. \\
& \left.+j z \sqrt{\left.\frac{\pi b_{k}^{2} \bar{\gamma}_{k}}{L+\sum_{i=1}^{L} b_{i}^{2} \bar{\gamma}_{i}}\right]}\right\} z^{-1}
\end{aligned}
$$

where $\bar{\gamma}_{k}$ denotes the ASNR of the $k$ th branch, ${ }_{1} F_{1}(a ; b ; c)$ is the confluent hypergeometric function, the $\operatorname{Im}\{\cdot\}$ stands for the imaginary part of its argument, and $j=\sqrt{-1}$. Note that by using $[28,(7.1 .21),(13.6 .15)]$ and $[29,(9.2 .40)]$, the confluent hypergeometric function in (16) can be written in a simpler form as

$$
{ }_{1} F_{1}\left(-\frac{1}{2} ; \frac{1}{2} ; c\right)=\exp (c)-\sqrt{\pi c} \operatorname{erfi}(\sqrt{c})
$$

where $\operatorname{erfi}(\cdot)$ is the imaginary error function defined as $\operatorname{erfi}(x)=\operatorname{erf}(j x) / j$. Moreover, applying the GaussHermite's numerical integration [28, p. 924], also proposed in [6], (15) can be written as

$$
P_{e}=\frac{1}{2}-\frac{1}{\pi} \sum_{m=1}^{n} \omega_{m} H\left(z_{m}\right)
$$

where $2 n$ is the integration order, $z_{m}$ are the abscissas, and $\omega_{m}$ the weights factors, which are given in [28, Table 25.10]. Hence, the ASEP is expressed as a finite sum of functions, where the weights $w_{k}$ are the only unknown parameters and the optimization problem described by (14) can be solved by applying well-known numerical optimization methods, such as the effective and computationally compact Nelder-Mead method [30], included in the most well-known software packages (e.g., Mathematica).

Next, we facilitate the use of the proposed method through an example.

Example 1: We consider a BRC receiver with $L=4$ diversity branches, where the fading amplitudes $a_{k}$ are Rayleigh distributed RVs. The PDP is assumed to be $\bar{\gamma}_{k}=\bar{\gamma} / k, k=$ $1, \ldots, L$, where $\bar{\gamma}$ is the ASNR of the first diversity branch. Following are the steps that the proposed algorithm follows.

1) Estimation of the ASNR at the $k$ th diversity $\bar{\gamma}_{k}$ yielding the vector $\bar{\gamma}=\left[\begin{array}{llll}10 & 6.9 & 5.2 & 4\end{array}\right] \mathrm{dB}$.

2) For a given integration order $2 n$ of the Hermite's integration rule, the $P_{e}$ in (18) is formed. The abscissas $z_{m}$ and the weights factors $\omega_{m}$ are given in [28, Table 25.10].

3) The optimization algorithm is utilized to find the vector $\Omega$ that solves the minimization problem (14). The vector $\Omega$ is found to be $\boldsymbol{\Omega}=\left[\begin{array}{llll}1 & 0.95 & 0.9 & 0.87\end{array}\right]$.

A Special Case: $L=3$ - In this case, the ASEP is given by the closed-form expression [7]

$$
\begin{aligned}
P_{e}(\mathbf{w})= & \frac{1}{2}-\mathcal{F}\left(\bar{\gamma}_{1}^{\prime}, \bar{\gamma}_{2}^{\prime}, \bar{\gamma}_{3}^{\prime}, 3 \kappa\right)-\mathcal{F}\left(\bar{\gamma}_{2}^{\prime}, \bar{\gamma}_{3}^{\prime}, \bar{\gamma}_{1}^{\prime}, 3 \kappa\right) \\
& -\mathcal{F}\left(\bar{\gamma}_{3}^{\prime}, \bar{\gamma}_{1}^{\prime}, \bar{\gamma}_{2}^{\prime}, 3 \kappa\right)+\frac{\pi}{4} \sqrt{\frac{\bar{\gamma}_{1}^{\prime} \bar{\gamma}_{2}^{\prime} \bar{\gamma}_{3}^{\prime}}{\left(\bar{\gamma}_{1}^{\prime}+\bar{\gamma}_{2}^{\prime}+\bar{\gamma}_{3}^{\prime}+3 \kappa\right)^{3}}}
\end{aligned}
$$

where $\bar{\gamma}_{k}^{\prime}=b_{k}^{2} \bar{\gamma}_{k}$ and the constant $\kappa$ equals 1 for BPSK. The function $\mathcal{F}$ is defined as

$$
\begin{aligned}
\mathcal{F}(x, y, z, c)= & \frac{1}{2} \sqrt{\frac{x(x+y+c)(x+z+c)}{(x+y+z+c)^{3}}} \\
& \times{ }_{2} F_{1}\left(-\frac{1}{2},-\frac{1}{2} ; \frac{1}{2} ; \frac{y z}{(x+y+c)(x+z+c)}\right)
\end{aligned}
$$

with ${ }_{2} F_{1}$ denoting the Gauss hypergeometric function. Using [28, (15.3.3), (15.2.12), (15.1.6)]

$$
{ }_{2} F_{1}\left(-\frac{1}{2},-\frac{1}{2} ; \frac{1}{2} ; v\right)=\sqrt{v} \sin ^{-1}(\sqrt{v})+\sqrt{1-v}
$$

and (20) can be reduced to the expression that contains only elementary functions

$$
\begin{aligned}
\mathcal{F}(x, y, z, c)= & \frac{1}{2} \sqrt{\frac{x(x+y+c)(x+z+c)}{(x+y+z+c)^{3}}} \\
& \times \sqrt{v} \sin ^{-1}(\sqrt{v})+\sqrt{1-v}
\end{aligned}
$$

where $v=y z /(x+y+c)(x+z+c)$. Note that (22) is much simpler than the corresponding expression given in [7], and results in lower complexity and shorter computational time for the minimization problem.

2) Nakagami-M Fading: For Nakagami- $m$ fading channels, the ASEP can be obtained by [8]

$$
P_{e}=\frac{2}{\pi} \int_{0}^{\pi / 2} \frac{\Psi(\tan z)}{\sin (2 z)} d z
$$

with

$$
\Psi(\omega)=\operatorname{Re}\left\{\omega G(\omega) \varphi_{x}^{*}(\omega)\right\}
$$

where $G(\omega)$ depends on the modulation scheme and the notation $\varphi_{x}^{*}(\omega)$ denotes the complex conjugate of the characteristic function $(\mathrm{CHF})$, which is given by

$$
\varphi_{x}(\omega)=\prod_{k=1}^{L} \varphi_{x_{k}}\left(\frac{\omega}{\sqrt{L}}\right)
$$

with

$$
\begin{aligned}
\varphi_{x_{k}}(\omega)= & { }_{1} F_{1}\left(m_{k} ; \frac{1}{2} ; \frac{-b_{k}^{2} \bar{\gamma}_{k} \omega^{2}}{4 m_{k}}\right)+j \omega \frac{\Gamma\left(m_{k}+(1 / 2)\right)}{\Gamma\left(m_{k}\right)} \\
& \times \sqrt{\frac{b_{k}^{2} \bar{\gamma}_{k}}{m_{k}}}{ }_{1} F_{1}\left(m_{k}+\frac{1}{2} ; \frac{3}{2} ; \frac{-b_{k}^{2} \bar{\gamma}_{k} \omega^{2}}{4 m_{k}}\right)
\end{aligned}
$$

where $m_{k}$ is the fading parameter of the $k$ th branch, which ranges from 0.5 to infinity. Using the Gauss-Kronrod quadrature numerical integration method [31], which approximates the value of an integral as a linear combination of values of the integrand evaluated at specific points, i.e.,

$$
\int_{a}^{b} f(z) d z=\sum_{m=1}^{n} \omega_{m} f\left(z_{m}\right)
$$


where $2 n$ is the integration order, $z_{m}$ are the abscissas, and $\omega_{m}$ the weight factors, (23) can be written as

$$
P_{e}=\frac{2}{\pi} \sum_{m=1}^{n} \omega_{m} \frac{\Psi\left(z_{m}\right)}{\sin \left(z_{m}\right)}
$$

and the optimization problem that is formed can be solved similarly as for the Rayleigh case.

For the case of binary modulation schemes [e.g., continuous phase-shift keying (CPSK) and continuous frequency-shift keying (CFSK)], $G(\omega)$ is given by [8]

$$
G(\omega)=\frac{a}{\omega}\left\{\frac{2}{\sqrt{\pi}} F\left(\frac{\omega}{2 \sqrt{b}}\right)+j\left[1-\exp \left(\frac{-\omega^{2}}{4 b}\right)\right]\right\}
$$

where the constants $a$ and $b$ depend on the modulation scheme and $F(\cdot)$ denotes the Dawson's integral:

$$
F(x)=\exp \left(-x^{2}\right) \int_{0}^{x} \exp \left(t^{2}\right) d t=x_{1} F_{1}\left(1, \frac{3}{2},-x^{2}\right) .
$$

For the general case of the $M$-PSK modulation, $G(\omega)$ is expressed as [8]

$$
\begin{aligned}
G(\omega)= & \frac{1}{\sqrt{\pi \omega}}\left\{F\left(\frac{\omega}{2 \sqrt{\kappa_{2}}}\right)\right. \\
& \left.-\exp \left(\frac{-\omega^{2} \sin ^{2}(\eta)}{4 \kappa_{2}}\right) F\left(\frac{\omega \cos (\eta)}{2 \sqrt{\kappa_{2}}}\right)\right\} \\
& +\frac{j \omega}{2 \pi} \int_{0}^{\eta} \frac{\sin ^{2}(\theta)}{\kappa_{2}}{ }_{1} F_{1}\left(1, \frac{3}{2}, \frac{-\omega^{2} \sin ^{2}(\eta)}{4 \kappa_{2}}\right) d \theta
\end{aligned}
$$

where $\eta=\pi-\pi / M$ and $\kappa_{2}=\sin ^{2}(\pi / M)$. Note that the confluent hypergeometric function in (29) and (30) can be written here as [28, eq. (7.1.21)]

$$
{ }_{1} F_{1}\left(1 ; \frac{3}{2} ; c\right)=\frac{\exp (c) \sqrt{\pi}}{2 \sqrt{c}} \operatorname{erf}(\sqrt{c}) .
$$

Next, the proposed method is illustrated through an example.

Example 2: We consider a BRC receiver with $L=4$ diversity branches, where the fading amplitudes $a_{k}$ are Nakagami- $m$ distributed RVs. The PDP is assumed to be exponential, i.e., $\bar{\gamma}_{k}=\bar{\gamma} e^{-d(k-1)}, k=1, \ldots, L$, where $d$ is the decaying factor. The implementation of the proposed algorithm can be summarized again in the following three steps.

1) Estimation of the ASNR at the $k$ th diversity $\bar{\gamma}_{k}$ and the fading parameters $m_{k}$ yielding the vectors $\bar{\gamma}=$ $\left[\begin{array}{llll}15 & 10.6 & 6.3 & 2\end{array}\right] \mathrm{dB}$ and $m=\left[\begin{array}{llll}1.5 & 1.24 & 2.95 & 1.29\end{array}\right]$.

2) For a given integration order $2 n$ of the Gauss-Kronrod quadrature method, the $P_{e}$ in (27) is formed. The abscissas $z_{m}$ and the weights factors $\omega_{m}$ are given in tables or can be produced by software packages such as Mathematica.

3) The optimization algorithm is utilized to find the vector $\Omega$ that solves the minimization problem (14). The vector $\boldsymbol{\Omega}$ is found to be $\boldsymbol{\Omega}=\left[\begin{array}{llll}0.79 & 0.71 & 0.99 & 0.61\end{array}\right]$.

\section{B. Maximization of the Combined ASNR}

Apart from the ASEP, other performance criteria such as the outage probability (OP) or the combined ASNR could also be considered in order to derive the optimum weights for the BRC receiver. The formulation of an optimization problem similar to (14) would also require analytical expressions for these criteria. For the case of EGC operating over Nakagami- $m$ fading with nonidentically distributed paths, the OP can be found in analytical form in $[4,(9.186)]$ and [12]. In the former, the OP expression is based on the moment generating function (MGF) approach and relies on an algorithm for numerically inverting Laplace transforms of cumulative distribution functions (cdfs), while in the latter, it is based in the Padé approximants theory. However, both formulas involve an overall error term, and therefore, the utilization of the OP would not substantially simplify the minimization problem of (14).

In contrast to ASEP and OP, the ASNR is typically the easiest to evaluate performance measure and often serves as an excellent indicator of the overall fidelity of the system. However, it cannot be efficiently associated with the error performance, especially in diversity systems operating over fading channels, because the variance of the instantaneous SNR is totally ignored. Next, we examine the maximization of the combined ASNR in order to derive optimal weights for the BRC receiver. The combined ASNR of the BRC can be expressed as

$$
\bar{\gamma}_{\mathrm{BRC}}=\int_{0}^{\infty} \gamma_{\mathrm{BRC}} p_{\gamma_{\mathrm{BRC}}}(x) d x .
$$

The problem is to find the optimum weights $w_{k}$ per branch, or the vector $\boldsymbol{\Omega}=\left[\begin{array}{llll}\Omega_{1} & \Omega_{2} & \cdots & \Omega_{k}\end{array}\right]$, that depend only on the channel's statistics and maximize the average combined SNR, i.e.,

$$
\begin{aligned}
& \boldsymbol{\Omega}=\underset{\mathbf{w}}{\arg \max }\left\{\bar{\gamma}_{\mathrm{BRC}}\right\} \\
\text { s.t. } \quad & w_{k} \in\left(l_{1}, l_{2}\right) .
\end{aligned}
$$

The EGC combined ASNR for independent Nakagami- $m$ fading is given in closed form as [12] ${ }^{1}$

$$
\bar{\gamma}_{\mathrm{EGC}}=\frac{\Gamma(m+1)}{\Gamma(m) m L} \sum_{i=1}^{L} \bar{\gamma}_{i}+\frac{\Gamma^{2}(m+(1 / 2))}{\Gamma^{2}(m) m L} \sum_{i, j=1, i \neq j}^{L} \sqrt{\overline{\bar{\gamma}}_{i} \bar{\gamma}_{j}} .
$$

Hence, the combined ASNR at the BRC output will be expressed as

$$
\begin{aligned}
\bar{\gamma}_{\mathrm{BRC}}= & \frac{\Gamma(m+1)}{\Gamma(m) m L} \sum_{i=1}^{L} b_{i}^{2} \bar{\gamma}_{i} \\
& +\frac{\Gamma^{2}\left(m+\frac{1}{2}\right)}{\Gamma^{2}(m) m L} \sum_{i, j=1, i \neq j}^{L} b_{i} b_{j} \sqrt{\bar{\gamma}_{i} \bar{\gamma}_{j}}
\end{aligned}
$$

which, for the case of Rayleigh fading $(m=1)$, reduces to

$$
\bar{\gamma}_{\mathrm{BRC}}=\frac{1}{L}\left(\sum_{i=1}^{L} b_{i}^{2} \bar{\gamma}_{i}+\frac{\pi}{4} \sum_{i, j=1, i \neq j}^{L} b_{i} b_{j} \sqrt{\bar{\gamma}_{i} \bar{\gamma}_{j}}\right) .
$$

The solution of (32) can be extracted by applying the NelderMead optimization method. With regard to the complexity of the

\footnotetext{
${ }^{1}$ Equation (33) corrects a typing error of [12, eq. (19)].
} 
function that has to be optimized, the ASNR criterion yields an easier optimization problem compared to the ASEP criterion. As will be discussed later in Section V, under certain fading conditions the ASNR can replace the ASEP criterion regarding the calculation of the BRC weights.

\section{LOW-COMPLEXITY BRC RECEIVERS}

The complexity of the BRC receiver that minimizes the ASEP can be significantly reduced - thus avoiding to solve the minimization problem described in (14) - by determining the optimal weights $w_{k}$ in terms of elementary functions of the average channels' parameters. As mentioned before, the weights $w_{k}$ depend on the channels' statistics (i.e., the ASNR of the $k$ th diversity path in Rayleigh fading or the ASNR and the fading parameter $m$ in Nakagami- $m$ fading), and therefore, could be expressed as a function of the channel's parameters as

$$
w_{k}=f_{k}(\bar{\gamma}), \quad k=1, \ldots, L
$$

for Rayleigh fading or

$$
w_{k}=f_{k}(\bar{\gamma}, \mathbf{m}), \quad k=1, \ldots, L
$$

for Nakagami- $m$ fading where $\bar{\gamma}=\left[\begin{array}{lll}\bar{\gamma}_{1} & \ldots & \bar{\gamma}_{k}\end{array}\right]$ and $\mathbf{m}=$ $\left[\begin{array}{lll}m_{1} & \cdots & m_{k}\end{array}\right]$. In order to find the optimal functions $f_{k}(\bar{\gamma})$ or $f_{k}(\bar{\gamma}, \mathbf{m})$, the minimization problem described in (14) can be written here as

$$
\boldsymbol{\Omega}=\underset{\mathbf{w}}{\arg \min }\left\{P_{e}\right\}
$$

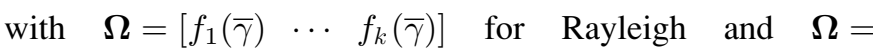
$\left[f_{1}(\bar{\gamma}, \mathbf{m}) \cdots f_{k}(\bar{\gamma}, \mathbf{m})\right]$ for Nakagami- $m$ fading channels. This problem is a nonlinear multiple function optimization problem, which is difficult to be solved analytically, but not impossible to derive an approximative solution numerically.

\section{A. Exponential PDP}

It is possible to find simple and accurate closed-form solutions for $f_{k}(\bar{\gamma})$ or $f_{k}(\bar{\gamma}, \mathbf{m})$, when exponential PDP is assumed, by applying nonlinear regression methods [32]. ${ }^{2}$ The exponential PDP model is encountered in most practical applications and also adopted by the European Telecommunications Standards Institute (ETSI) in the Universal Mobile Telecommunications System (UMTS) standards for the spacial channel model [33].

1) Rayleigh Fading: For the case of Rayleigh fading channels, the ASEP depends only on the ASNR of each branch. By applying the nonlinear regression method, the weight of the $k$ th branch for $M$-PSK modulation can be accurately approximated by the simple elementary function

$$
\Omega_{k}=\sqrt{1-\exp \left(-\frac{\bar{\gamma}_{k}}{M-1}\right)}
$$

where $M$ is the order of the $M$-PSK modulation. As is observed, only the first moment of the SNR at each branch is required in order to accurately approximate the optimum weights.

\footnotetext{
${ }^{2}$ This procedure was not applied for the maximization problem described in (32), since the extracted weights could not yield a better error performance, compared to the case of the ASEP's minimization.
}

2) Nakagami-m Fading: For the case of the Nakagami-m generalized fading model, the unknown function depends on both the ASNRs and the fading parameters $m_{k}$. In this case, the weight of the $k$ th branch can be accurately approximated by the following function:

$$
\Omega_{k}=\sqrt{\left(1-\exp \left(-\frac{\bar{\gamma}_{k}}{M-1}\right)\right)\left(1-\exp \left(-m_{k}\right)\right)} .
$$

In a real wireless system, both the ASNR and the parameter $m$ can be easily estimated and a large number of papers in the literature have dealt with this problem (see [34]-[36], and references therein).

3) The Case of Large Decay Factor: It can be verified easily that, for large values of the decay factor $d$, the branch with the highest mean SNR dominates and the MRC reduces to a single-channel receiver with an output SNR

$$
\gamma_{\mathrm{MRC}} \simeq \gamma_{1}
$$

Similarly, the combined output SNR of the EGC will be

$$
\gamma_{\mathrm{EGC}} \simeq \frac{\gamma_{1}}{L}
$$

On the other hand, when the weights of (39) and (40) are utilized, the output SNR of the BRC receiver is described as shown in (8). However, for $d \gg 1$ and $L>2, \sum_{k=1}^{L} \Omega_{k}^{2} \simeq 1$ and (8) is approximately equal to

$$
\gamma_{\mathrm{BRC}} \simeq\left(\sum_{k=1}^{L} \Omega_{k} \sqrt{\gamma_{k}}\right)^{2}
$$

Expanding the previous squared sum, we can write (41) as

$$
\begin{aligned}
\gamma_{\mathrm{BRC}}= & \Omega_{1} \sqrt{\gamma_{1}}\left(\Omega_{1} \sqrt{\gamma_{1}}\right)+\Omega_{2} \sqrt{\gamma_{2}}\left(2 \Omega_{1} \sqrt{\gamma_{1}}+\Omega_{2} \sqrt{\gamma_{2}}\right) \\
& +\Omega_{3} \sqrt{\gamma_{3}}\left(2 \Omega_{1} \sqrt{\gamma_{1}}+2 \Omega_{2} \sqrt{\gamma_{2}}+\Omega_{3} \sqrt{\gamma_{3}}\right)+\cdots
\end{aligned}
$$

Without loss of generality, for large values of the decay factor and assuming BPSK modulation, the weights $w_{k}$ can be written as

$$
\Omega_{k}=\sqrt{1-\exp \left(-\bar{\gamma}_{1} \exp (-d(k-1))\right)} \simeq 0, \quad k=2, \ldots, L
$$

which means that the weights of the $k$ th branch $(k \geq 2)$ will be almost null for both the Rayleigh and Nakagami- $m$ cases. Thus, (42) will be simplified to

$$
\begin{aligned}
\gamma_{\mathrm{BRC}} & =\Omega_{1} \sqrt{\gamma_{1}}\left(\Omega_{1} \sqrt{\gamma_{1}}\right)=\Omega_{1}^{2} \gamma_{1} \\
& =\left(1-\exp \left(-\bar{\gamma}_{1}\right)\right) \gamma_{1} \approx \gamma_{\mathrm{MRC}}
\end{aligned}
$$

for $\bar{\gamma}_{1}>0 \mathrm{~dB}$, which denotes that, for large decay factors, the instantaneous output $\mathrm{SNR}$ of the BRC receiver approximates that of the MRC receiver.

\section{B. Arbitrary PDP}

Next, we show by using the majorization theory [25] that although the approximated weights of (39) and (40) were derived assuming exponential PDP, they can also be used efficiently for 


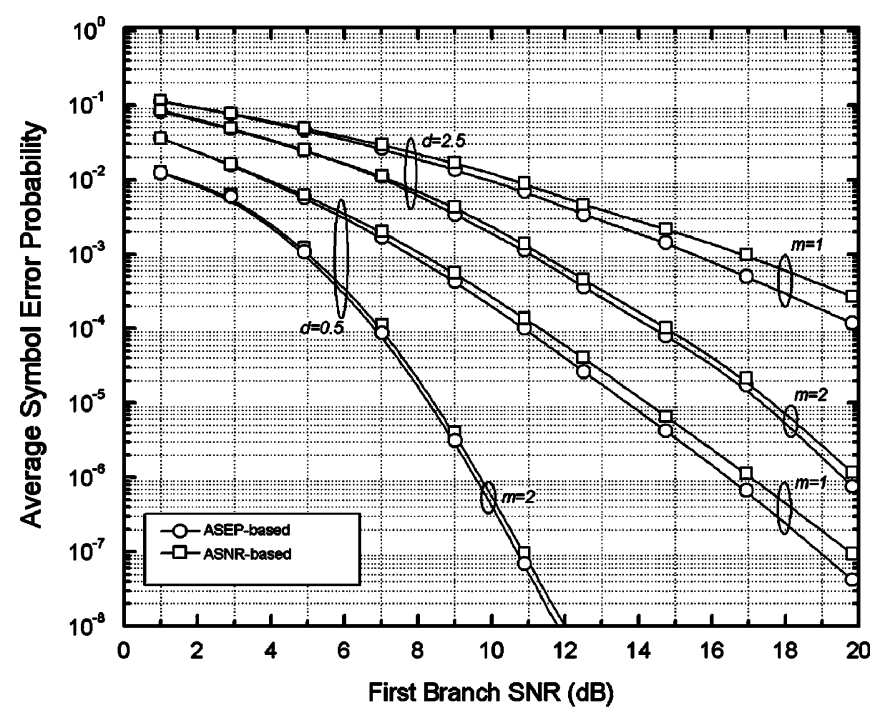

Fig. 2. ASEP of a BRC receiver $(L=5)$ operating over Nakagami- $m$ fading with exponential PDP when the weights are extracted by minimizing/ maximizing the ASEP/ASNR.

arbitrary PDPs. We note that the most important definitions of the majorization theory is sufficiently reviewed in [37].

Theorem 1: The ASEP $P_{e}^{\mathrm{EGC}}$ of an EGC receiver that operates in independent Rayleigh fading channels is an upper bound of the ASEP $P_{e}^{\mathrm{BRC}}$ of a BRC receiver that utilizes the weights given by (39) and operates in the same fading conditions, i.e., $P_{e}^{\mathrm{BRC}} \leq P_{e}^{\mathrm{EGC}}$, with the equality to hold for i.i.d. channels.

Proof: For proof, see the Appendix.

Theorem 1 also holds for Nakagami- $m$ fading channels with the same fading parameters. However, the proof is almost the same as before and omitted for the sake of brevity.

\section{COMPARISON WITH EGC, MRC, AND GSC RECEIVERS}

In this section, the performance of the proposed BRC receiver is compared with the conventional EGC, MRC, and GSC receivers. As mentioned before, BRC constitutes a virtual EGC receiver, and consequently, its performance analysis will not differ from the latter. Therefore, the BRC performance can be evaluated using already well-known results in the literature for the EGC receivers [3], [5], [6], [12].

\section{A. Average Symbol Error Probability (ASEP)}

In Fig. 2, we examine and compare the performance of the $B R C$ receiver that applies the ASNR criterion with that of the BRC that minimizes the ASEP, assuming BPSK modulation. The first observation that can be made concerns the impact of the Nakagami- $m$ parameter on the performance of the examined schemes. As the fading severity decreases (i.e., $m$ increases), the performance of the BRC that applies the ASNR criterion resembles that of the BRC that utilizes the ASEP criterion. Specifically, the difference can be as large as $1 \mathrm{~dB}$ for $m=1$, while it does not exceed $0.2 \mathrm{~dB}$ for $m=2$. The same observation can be made regarding the effect of the decaying factor on the error performance. Similar results can be extracted when employing different order of the $M$-PSK modulation or differ-
TABLE I

Optimum Weights $\Omega_{\text {opt }}$ Compared to the Weights $\Omega_{\text {app }}$ Given by (39) AND (40)

\begin{tabular}{|c|c|c|}
\hline & Rayleigh & Nakagami- $m$ \\
\hline $\bar{\gamma}_{1}$ & $\begin{array}{l}\Omega_{o p t} \\
\Omega_{a p p}\end{array}$ & $\begin{array}{l}\Omega_{o p t} \\
\Omega_{a p p}\end{array}$ \\
\hline \multirow[t]{2}{*}{0} & {$\left[\begin{array}{lllll}0.79 & 0.58 & 0.38 & 0.24 & 0.14\end{array}\right]$} & {$\left[\begin{array}{lllll}0.71 & 0.50 & 0.29 & 0.19 & 0.11\end{array}\right]$} \\
\hline & {$\left[\begin{array}{lllll}0.79 & 0.55 & 0.35 & 0.22 & 0.13\end{array}\right]$} & {$\left[\begin{array}{lllll}0.71 & 0.50 & 0.26 & 0.19 & 0.11\end{array}\right]$} \\
\hline \multirow[t]{2}{*}{10} & {$\left[\begin{array}{llllll}0.99 & 0.92 & 0.77 & 0.59 & 0.40\end{array}\right]$} & {$\left[\begin{array}{llllll}0.88 & 0.88 & 0.59 & 0.52 & 0.31\end{array}\right]$} \\
\hline & {$\left[\begin{array}{llllll}0.99 & 0.98 & 0.86 & 0.62 & 0.41\end{array}\right]$} & {$\left[\begin{array}{llllll}0.89 & 0.89 & 0.63 & 0.54 & 0.32\end{array}\right]$} \\
\hline \multirow[t]{3}{*}{15} & {$\left[\begin{array}{llllll}1 & 0.94 & 0.84 & 0.72 & 0.55\end{array}\right]$} & {$\left[\begin{array}{llllll}0.88 & 0.89 & 0.68 & 0.71 & 0.49\end{array}\right]$} \\
\hline & {$\left[\begin{array}{lllll}1 & 0.99 & 0.99 & 0.89 & 0.66\end{array}\right]$} & {$\left[\begin{array}{llllll}0.89 & 0.90 & 0.73 & 0.77 & 0.52\end{array}\right]$} \\
\hline & \multicolumn{2}{|c|}{$d=2$} \\
\hline \multirow[t]{2}{*}{0} & {$\left[\begin{array}{llllll}0.79 & 0.38 & 0.14 & 0.055 & 0.02\end{array}\right]$} & {$\left[\begin{array}{lllll}0.71 & 0.32 & 0.11 & 0.044 & 0.015\end{array}\right]$} \\
\hline & {$\left[\begin{array}{lllll}0.79 & 0.35 & 0.13 & 0.049 & 0.018\end{array}\right]$} & 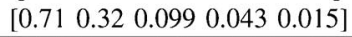 \\
\hline \multirow[t]{2}{*}{10} & {$\left[\begin{array}{llllll}0.99 & 0.80 & 0.40 & 0.15 & 0.058\end{array}\right]$} & {$\left[\begin{array}{llllll}0.89 & 0.66 & 0.27 & 0.12 & 0.042\end{array}\right]$} \\
\hline & {$\left[\begin{array}{lllll}0.99 & 0.86 & 0.41 & 0.15 & 0.057\end{array}\right]$} & {$\left[\begin{array}{lllll}0.898 & 0.78 & 0.30 & 0.13 & 0.046\end{array}\right]$} \\
\hline \multirow[t]{2}{*}{15} & {$\left[\begin{array}{llllll}0.99 & 0.93 & 0.60 & 0.26 & 0.099\end{array}\right]$} & {$\left[\begin{array}{llllll}0.90 & 0.81 & 0.43 & 0.21 & 0.072\end{array}\right]$} \\
\hline & {$\left[\begin{array}{lllll}1 & 0.99 & 0.66 & 0.27 & 0.10\end{array}\right]$} & {$\left[\begin{array}{llllll}0.89 & 0.90 & 0.48 & 0.23 & 0.08\end{array}\right]$} \\
\hline
\end{tabular}

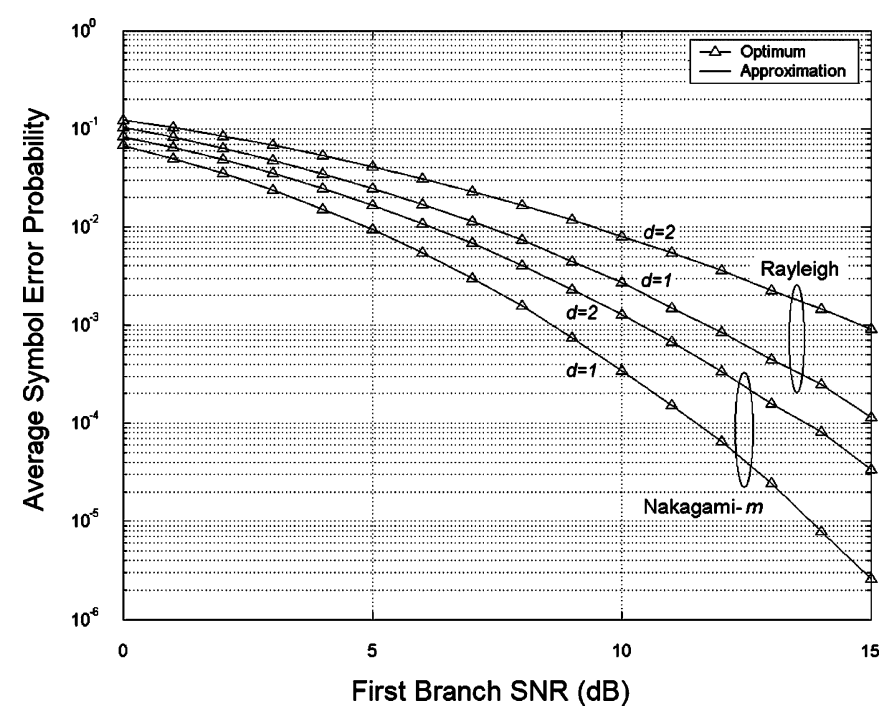

Fig. 3. ASEP of a BRC receiver $(L=6)$ operating over Rayleigh or Nakagami- $m$ fading with exponential PDP when the optimum or the approximated weights are used.

ent number of diversity branches. Thereby, under certain fading conditions - as described before- the utilization of the ASNR criterion for the calculation of the BRC weights can be considered as an interesting and efficient solution, which can replace the ASEP criterion.

It is obvious that the approximation of the optimum weights by the elementary functions, given by (39) and (40), is of great practical interest, since it leads to a receiver with low complexity, almost equivalent to that of $E G C$. Next, we examine the accuracy of this approximation and its effect on the ASEP.

In Table I, the optimum weights derived by solving the minimization problem in (14) are compared to the weights given by (39) and (40) for Rayleigh and Nakagami- $m$ fading channels with exponential PDP and BPSK modulation. From this table, it is evident that the approximated weights are very close to the optimum ones. For the same example, the ASEP is plotted in Fig. 3, using both the optimal and the approximated weights. 


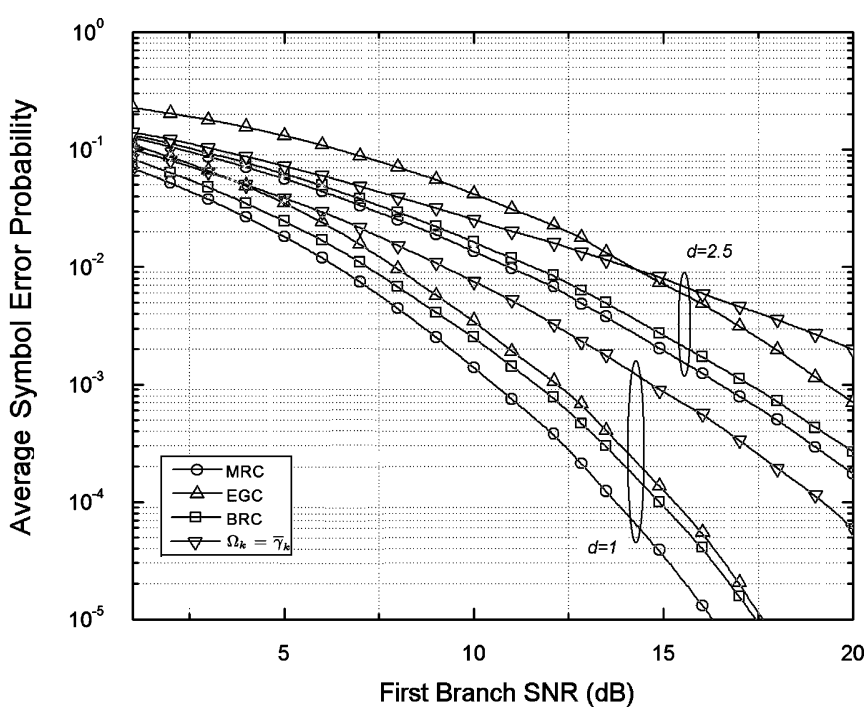

Fig. 4. ASEP of a BRC receiver $(L=5)$ operating over Rayleigh fading with exponential PDP, when $\Omega_{k}=\bar{\gamma}_{k}$ and $\Omega_{k}=\sqrt{1-\exp \left(-\bar{\gamma}_{k}\right)}$ (BPSK modulation)

It is easily observed that, for the specific example, the approximation does not affect the ASEP, i.e., the procedure to solve the minimization problem described in (14) could be avoided, resulting in low-complexity practical receivers. Similar results concerning the efficiency of the approximation can be obtained for different order of the $M$-PSK modulation, values of the $m$ parameter or decaying factors. In the following, the BRC receiver utilizes the approximated weights.

One might think of using the mean SNR of each branch as a simple weighting method regardless of the fading distribution. However, this method cannot constitute an efficient weighting method for BRC, since Theorem 1 does not hold for this case. In other words, by applying the mean SNR as a weighting method, an improved performance over EGC cannot be guaranteed; instead, the performance is most likely to degrade. This method is compared against the BRC method in Fig. 4.

As shown in Section IV, the approximated weights can also be applied when the PDP is not necessarily exponential. This is evident in Fig. 5 where the error performance of the MRC, EGC, and BRC is plotted, when an arbitrary PDP is assumed. In the specific example, the PDP is assumed to be $\bar{\gamma}_{k}=\bar{\gamma}_{1}[1,0.01,0.7,0.3,0.2]$.

Since BRC is proposed as an alternative diversity scheme, with an error performance between MRC and EGC, it would be interesting to compare it with the GSC receiver. Fig. 6 depicts the error performance of the MRC, the EGC, the BRC, and the GSC receivers in Nakagami- $m$ fading with $m=3$ and exponential PDP, when BPSK modulation is used. Although this comparison is not fair, because GSC requires the estimation of the instantaneous channel gains, it can be easily observed that BRC may outperform GSC in certain conditions. Moreover, when $d$ is large (as it was also shown in Section IV), the performance of BRC approaches that of MRC.

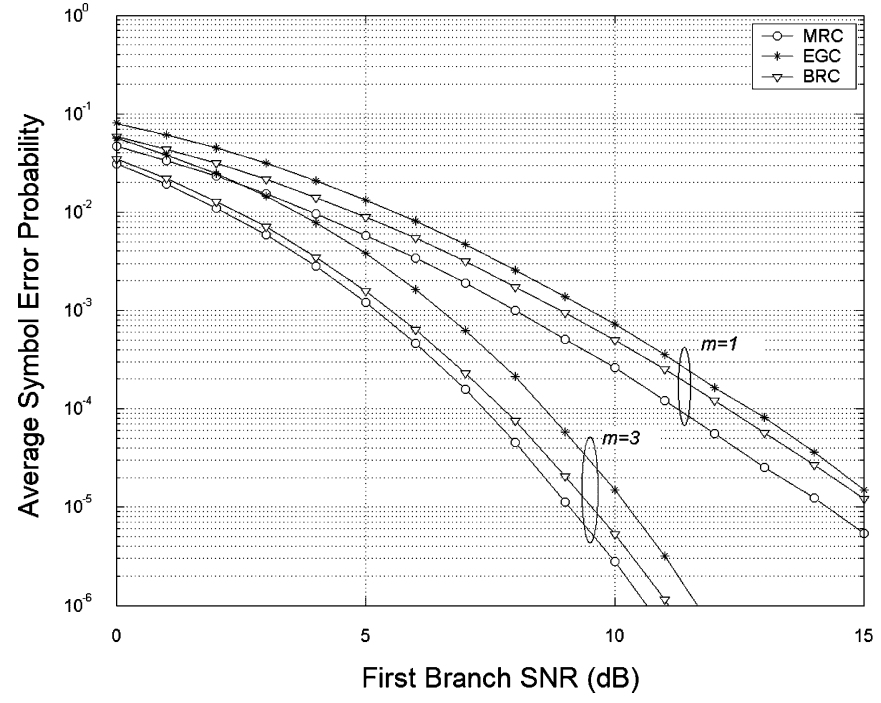

Fig. 5. ASEP of MRC, EGC, and BRC receivers in Nakagami- $m$ fading with arbitrary PDP (BPSK modulation).

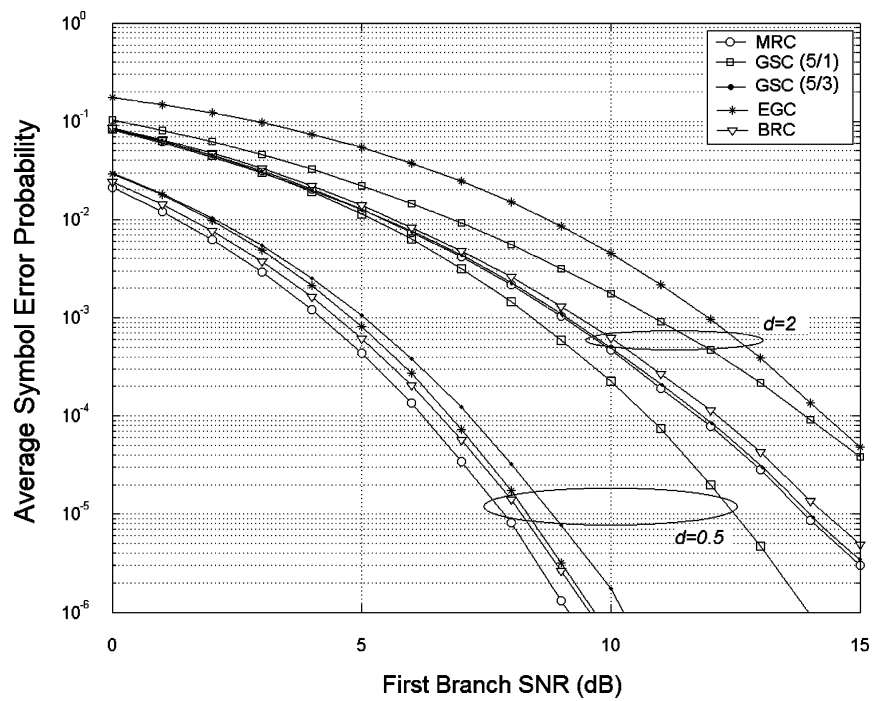

Fig. 6. Impact of the decay factor in MRC, EGC, and BRC receivers in Nakagami- $m$ fading $(m=3)$ with exponential PDP and $L=5$, using BPSK modulation.

\section{B. Combined ASNR}

The average combined SNR of the MRC, EGC (33), and BRC (34) receivers is compared in Fig. 7 for Rayleigh fading and exponential PDP with two different decay factors. We observe that, in the case of the EGC, the addition of a diversity branch does not always increase the normalized average output SNR due to combining loss; the weak branches contribute more in increasing the noise than the signal power. On the contrary, in $\mathrm{BRC}$, the addition of weak branches does not increase the noise because they are optimally weighted. For example, if a branch is very weak (i.e., its SNR is very low), its weight will also be low, which means that the noise of this branch will be suppressed and the combining loss will be almost eliminated. 


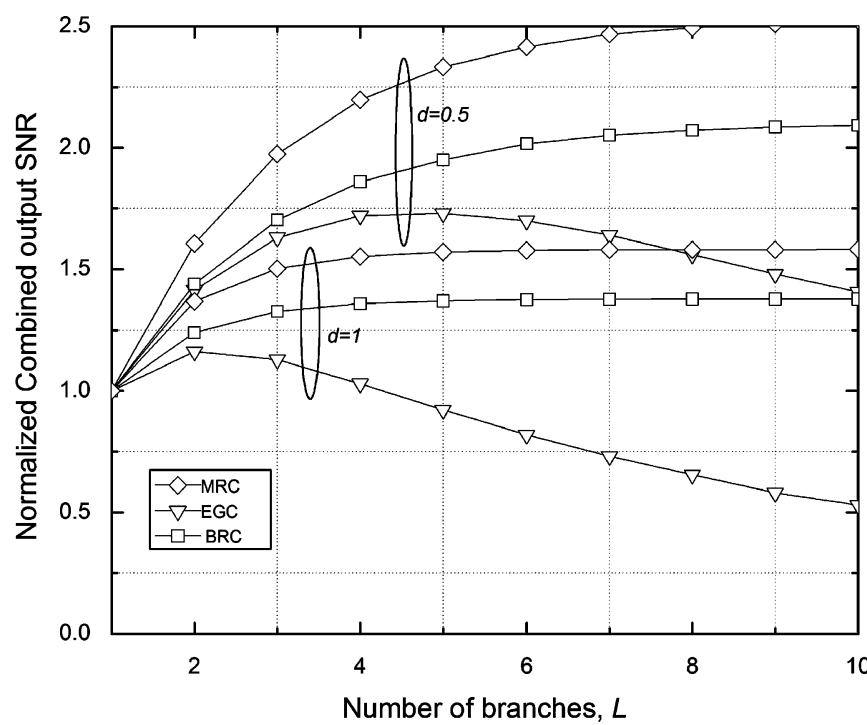

Fig. 7. Normalized output ASNR $\bar{\gamma}_{\text {out }} / \bar{\gamma}_{1}$ of the MRC, EGC, and BRC receivers in Rayleigh fading with exponential PDP.

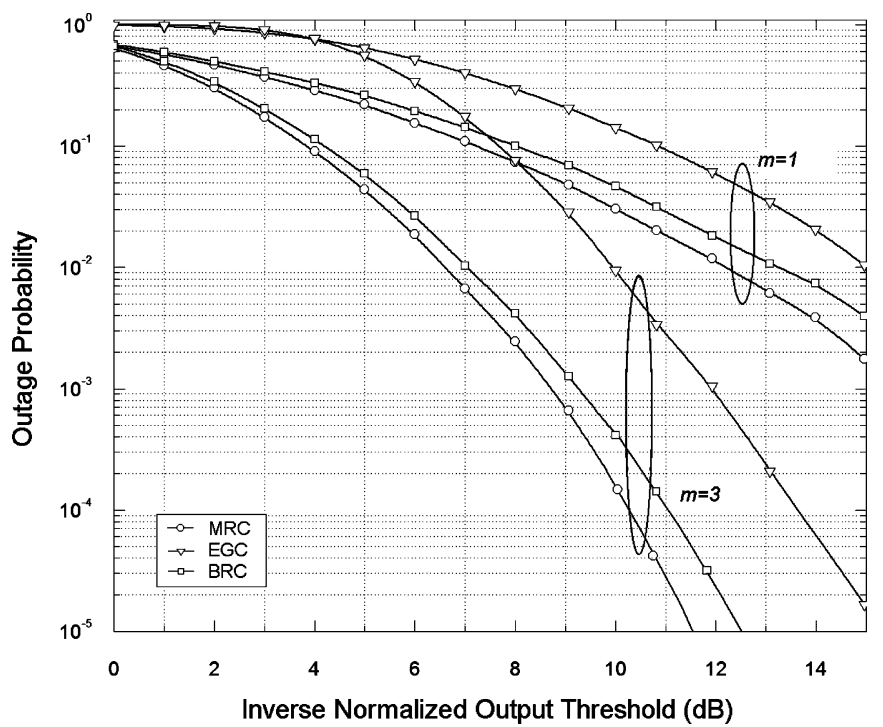

Fig. 8. OP of the MRC, EGC, and BRC receivers in Nakagami- $m$ fading, with exponential PDP $(d=1.5)$, using BPSK modulation.

\section{Outage Probability}

The OP of the BRC receivers can be evaluated by exploiting already published results for the EGC receivers [5], [12] and will be omitted in this paper. In Fig. 8, we compare the OP of the MRC, EGC, and BRC receivers in Nakagami- $m$ fading with exponential PDP. As is evident, the outage performance of the BRC is better than that of the EGC's for both fading parameters. Moreover, for better fading conditions (i.e., larger $m$ parameter), the outage performance of the former is closer to that of the MRC receiver.

In Fig. 9, where the impact of the decay factor on the OP is examined, we observe that the performance of the BRC is close to that of the MRC, in contrast to the EGC whose performance is dramatically decreased as $d$ increases.

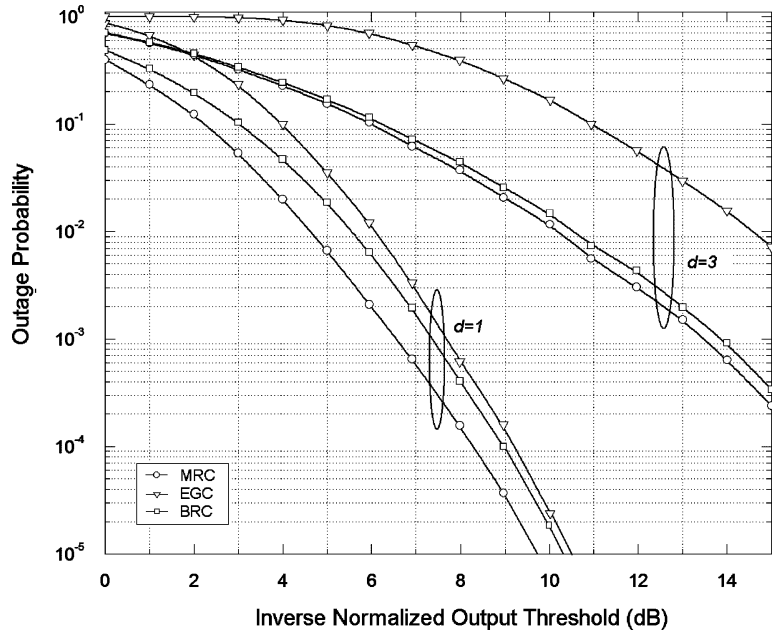

Fig. 9. OP of the MRC, EGC, and BRC receivers in Nakagami- $m$ fading $(m=2)$ with exponential PDP, using BPSK modulation.

We must also note that despite the fact that BRC is designed to minimize the ASEP-as expected-it also improves other performance metrics, such as the combined ASNR and the OP.

\section{Impact of Imperfect Channel Statistics Estimation}

As mentioned before, the BRC receiver requires the knowledge of the average fading power and (for the case of Nakagami- $m$ fading) the $m$-parameters of each branch, the estimation of which has been extensively studied in the literature [34]-[36]. Many practical and efficient methods have been proposed, but an accurate and perfect estimation of the fading parameters may not always be possible. We have investigated the $\mathrm{BRC}$ performance when both the average fading power and the Nakagami- $m$ parameter were estimated imperfectly. Detailed results are omitted for the sake of brevity and only the final conclusions are presented as follows. Considering a maximum error of $20 \%$, i.e., $\bar{\gamma}_{k}^{e}= \pm 1.2 \bar{\gamma}_{k}$ and $m_{k}^{e}= \pm 1.2 m_{k}$, where $\bar{\gamma}_{k}^{e}$ and $m_{k}^{e}$ denote the estimated parameters and $\bar{\gamma}_{k}$ and $m_{k}$ the real ones at the $k$ th branch, the ratio of the ASEP to the real one (i.e., with no estimation errors) was not greater than 0.998 , i.e., ASEP/ASEP opt $\leq 0.998$. Moreover, it was observed that the performance of the $\mathrm{BRC}$ is more sensitive to the average fading power estimation errors than to the $m$-parameter estimation errors. However, the average fading power is typically the easiest to estimate measure using existing techniques.

\section{CONCLUSION}

A multichannel receiver called BRC was presented. It can be efficiently applied in multipath fading environments, with equal energy constellation schemes and coherent detection. Without utilizing continuous estimation of the channels' fading amplitudes, the diversity branches are optimally weighted by longterm channel's-statistics-based weights, which minimize the ASEP or maximize the combined ASNR. It was shown that the BRC receiver constitutes a virtual EGC receiver that includes the conventional EGC receiver as a special case when the weights are equal to unity. In the important practical case of 
exponential PDP, which is common in RAKE receivers modeling and is adopted for the UMTS standard, the optimum weights can be accurately approximated by simple elementary functions. Moreover, it was proved that the utilization of these weights ensures an error performance improvement over EGC for arbitrary PDPs, eliminating its main disadvantage, i.e., the combining loss. Moreover, for strong multipath environments, the performance of the BRC approaches that of MRC. Generally, the $\mathrm{BRC}$ receiver seems to be an attractive low-complexity scheme for wideband wireless communications systems, where a large number of diversity branches exist, due to the strong multipath effects and PDPs that characterize their operating environments.

\section{ACKNOWLEDGMENT}

The authors would like to thank Prof. L. Georgiadis, Mr. D. Michalopoulos, and Mr. M. Gatzianas, Aristotle University of Thessaloniki, for useful discussions on the optimization theory. They would also like to thank the Editor and the anonymous reviewers for their helpful comments and constructive suggestions that considerably improved the quality of this paper.

\section{APPENDIX}

\section{PROOF OF THEOREM 1}

Let the vector $\mathrm{g}_{\mathrm{EGC}}=\left[\bar{\gamma}_{1}, \ldots, \bar{\gamma}_{L}\right]$ denote the PDP of an $L$-branch EGC receiver, and without loss of generality, assume that $\bar{\gamma}_{1} \geq \bar{\gamma}_{2} \geq \cdots \geq \bar{\gamma}_{L}>0$. As mentioned in Section II, the $B R C$ receiver constitutes a virtual EGC receiver whereas the RV $\gamma_{k}$ is replaced by the RV $b_{k}^{2} \gamma_{k}$, and therefore, the PDP of the $\mathrm{BRC}$ will be denoted by the vector $\mathbf{g}_{\mathrm{BRC}}=\left[b_{1}^{2} \bar{\gamma}_{1}, \ldots, b_{L}^{2} \bar{\gamma}_{L}\right]$.

In order to prove that $P_{e}^{\mathrm{BRC}} \leq P_{e}^{\mathrm{EGC}}$, it is sufficient to prove that $\mathbf{g}_{\mathrm{EGC}} \prec_{w} \mathbf{g}_{\mathrm{BRC}}$, where $\mathbf{q}_{1} \prec_{w} \mathbf{q}_{2}$ stands for the weak majorization of $\mathbf{q}_{2}$ over $\mathbf{q}_{1}$ [37, Theorem 1 and Definition 4]. Thus, it has to be proved that

$$
\sum_{i=1}^{k}\left(b_{i}^{2} \bar{\gamma}_{i}\right) \geq \sum_{i=1}^{k}\left(\bar{\gamma}_{i}\right), \quad k=1, \ldots, L
$$

or

$$
\sum_{i=1}^{k}\left[\left(b_{i}^{2}-1\right) \bar{\gamma}_{i}\right] \geq 0
$$

Substituting the weights given by (39) in (46) and after simple manipulations, the earlier inequality can be expressed as follows:

$$
\begin{aligned}
& \frac{1}{L-\sum_{i=1}^{L} \exp \left(-\bar{\gamma}_{i}\right)} \\
& \sum_{i=1}^{k}\left[\left(\sum_{i=1}^{L} \exp \left(-\bar{\gamma}_{i}\right)-L \exp \left(-\bar{\gamma}_{i}\right)\right) \bar{\gamma}_{i}\right] \geq 0
\end{aligned}
$$

and since $L-\sum_{i=1}^{L} \exp \left(-\bar{\gamma}_{i}\right)>0,(47)$ reduces to

$$
\frac{1}{L}\left(\sum_{i=1}^{k} \bar{\gamma}_{i}\right)\left(\sum_{i=1}^{L} \exp \left(-\bar{\gamma}_{i}\right)\right)-\sum_{i=1}^{k}\left(\bar{\gamma}_{i} \exp \left(-\bar{\gamma}_{i}\right)\right) \geq 0
$$

or

$$
\begin{gathered}
\frac{1}{L}\left(\sum_{i=1}^{k} \bar{\gamma}_{i}\right)\left(\sum_{i=1}^{k} \exp \left(-\bar{\gamma}_{i}\right)+\sum_{i=k+1}^{L} \exp \left(-\bar{\gamma}_{i}\right)\right) \\
-\sum_{i=1}^{k}\left[\bar{\gamma}_{i} \exp \left(-\bar{\gamma}_{i}\right)\right] \geq 0 .
\end{gathered}
$$

Finally,

$$
\begin{gathered}
\left(\frac{1}{L} \sum_{i=1}^{k} \bar{\gamma}_{i} \sum_{i=1}^{k} \exp \left(-\bar{\gamma}_{i}\right)-\sum_{i=1}^{k} \bar{\gamma}_{i} \exp \left(-\bar{\gamma}_{i}\right)\right) \\
+\left(\frac{1}{L} \sum_{i=1}^{k} \bar{\gamma}_{i} \sum_{i=k+1}^{L} \exp \left(-\bar{\gamma}_{i}\right)\right) \geq 0
\end{gathered}
$$

which is true since the second term in (50) is positive and the first term is also positive according to the well-known Chebyshev's sum inequality, i.e.,

$$
\frac{1}{L} \sum_{i=1}^{L} A_{i} \sum_{i=1}^{L} B_{i}-\sum_{i=1}^{L} A_{i} B_{i} \geq 0
$$

under the condition that $A_{1} \geq A_{2} \geq \cdots \geq A_{L}$ and $B_{1} \leq B_{2}$ $\leq \cdots \leq B_{L}$.

\section{REFERENCES}

[1] A. Annamalai, C. Tellambura, and V. K. Bhargava, "Efficient computation of MRC diversity performance in Nakagami fading channel with arbitrary parameters," Electron. Lett., vol. 34, no. 12, pp. 1189-1190, Jun. 1998.

[2] V. A. Aalo, "Performance of maximal-ratio diversity systems in a correlated Nakagami-fading environment," IEEE Trans. Commun., vol. 43, no. 8, pp. 2360-2369, Aug. 1995.

[3] A. Annamalai, C. Tellambura, and V. K. Bhargava, "Exact evaluation of maximal-ratio and equal-gain diversity receivers for $M$-ary QAM on Nakagami fading channels," IEEE Trans. Commun., vol. 47, no. 9, pp. 1335-1344, Sep. 1999.

[4] M. K. Simon and M. Alouini, Digital Communication Over Fading Channels, 2nd ed. Hoboken, NJ: Wiley, 2005.

[5] N. C. Beaulieu and A. A. Abu-Dayya, "Analysis of equal gain diversity on Nakagami fading channels," IEEE Trans. Commun., vol. 39, no. 2 , pp. 225-234, Feb. 1991.

[6] Q. T. Zhang, "A simple approach to probability of error for equal gain combiners over Rayleigh channels," IEEE Trans. Veh. Technol., vol. 48 , no. 4, pp. 1151-1154, Jul. 1999.

[7] Q. T. Zhang, "Probability of error for equal-gain combiners over Rayleigh channels: Some closed-form solutions," IEEE Trans. Commun., vol. 45, no. 3, pp. 270-273, Mar. 1997.

[8] A. Annamalai, C. Tellambura, and V. K. Bhargava, "Equal-gain diversity receiver performance in wireless channels," IEEE Trans. Commun., vol. 48, no. 10, pp. 1732-1745, Oct. 2000.

[9] R. K. Mallik, M.Z. Win, and J. H. Winters, "Performance of dual-diversity EGC in correlated Rayleigh fading with unequal branch SNRs," IEEE Trans. Commun., vol. 50, no. 7, pp. 1041-1044, Jul. 2002.

[10] C. Iskander and P. T. Mathiopoulos, "Performance of dual-branch coherent equal-gain combining in correlated Nakagami- $m$ fading," Electron. Lett., vol. 39, no. 15, pp. 1152-1154, Jul. 2003.

[11] N. Sagias, "Closed-form analysis of equal-gain diversity in wireless radio networks," IEEE Trans. Veh. Technol., vol. 56, no. 1, pp. 173-182, Jan. 2007.

[12] G. K. Karagiannidis, "Moments-based approach to the performance analysis of equal gain diversity in Nakagami- $m$ fading," IEEE Trans. Commun., vol. 52, no. 5, pp. 685-690, May 2004.

[13] E. A. Neasmith and N. C. Beaulieu, "New results on selection diversity," IEEE Trans. Commun., vol. 46, no. 5, pp. 695-704, May 1998.

[14] M. K. Simon and M. Alouini, "A unified performance analysis of digital communications with dual selective combining diversity over correlated 
Rayleigh and Nakagami- $m$ fading channels," IEEE Trans. Commun., vol. 47, no. 1, pp. 33-43, Jan. 1999.

[15] A. A. Abu-Dayya and N. C. Beaulieu, "Analysis of switched diversity systems on generalized-fading channels," IEEE Trans. Commun., vol. 42, no. 11, pp. 2959-2966, Nov. 1994.

[16] Y. Ko, M. Alouini, and M. K. Simon, "Analysis and optimization of switched diversity systems," IEEE Trans. Veh. Technol., vol. 49, no. 5, pp. 1813-1831, Sep. 2000.

[17] N. C. Sagias and P. T. Mathiopoulos, "Switched diversity receivers over generalized gamma fading channels," IEEE Commun. Lett., vol. 9, no. 10, pp. 871-873, Oct. 2005.

[18] C. Tellambura, A. Annamalai, and V. K. Bhargava, "Unified analysis of switched diversity systems in independent and correlated fading channels," IEEE Trans. Commun., vol. 49, no. 11, pp. 1955-1965, Nov. 2001.

[19] T. Eng, N. Kong, and L. B. Milstein, "Comparison of diversity combining techniques for Rayleigh-fading channels," IEEE Trans. Wireless Commun., vol. 5, no. 8, pp. 2153-2163, Sep. 1996.

[20] J. C. Hancock and W. C. Lindsey, "Optimum performance of self-adaptive systems operating through a Rayleigh-fading medium," IEEE Trans. Commun. Syst., vol. 11, no. 4, pp. 443-453, Dec. 1963.

[21] G. L. Turin, "On optimal diversity reception," IRE Trans. Inf. Theory, vol. IT-7, pp. 154-167, Jul. 1961.

[22] G. L. Turin, "On optimal diversity reception II," IRE Trans. Commun. Syst., vol. CS-10, pp. 22-31, Mar. 1962.

[23] W. C. Lindsey and M. K. Simon, Telecommunications Systems Engineeriing. Upper Saddle River, NJ: Prentice-Hall, 1973.

[24] J. H. Kotecha and A. M. Sayeed, "Transmit signal design for optimal estimation of correlated MIMO channels," IEEE Trans. Signal Process., vol. 52, no. 2, pp. 546-557, Feb. 2004.

[25] A. W. Marshall and I. Olkin, Inequalities: Theory of Majorization and its Applications. New York: Academic, 1979.

[26] J. Foerster, "Channel modeling subcommittee report final," IEEE P802.15.3.a Working Group, Proposal V1.0, 2003.

[27] L. Xiao and X. Dong, "Unified analysis of generalized selection combining with normalized threshold test per branch," IEEE Trans. Commun., vol. 44, no. 9, pp. 1117-1129, Aug. 2006.

[28] M. Abramovitz and I. A. Stegun, Handbook of Mathematical Functions With Formulas, Graphs, and Mathematical Tables, 9th ed. New York: Dover, 1972.

[29] I. S. Gradshteyn and I. M. Ryzhik, Table of Integrals, Series, and Products, 6th ed. New York: Academic, 2000.

[30] J. A. Nelder and R. Mead, "A simplex method for function minimization," Comput. J., vol. 7, pp. 308-313, 1965.

[31] D. Calvetti, G. H. Golub, W. Gragg, and L. Reichel, "Computation of Gauss-Kronrod quadrature rules," Stanford University Scientific Computing/Computational Mathematics Rep. SCCM-98-09, 2000.

[32] G. A. Seber and C. J. Wild, Nonlinear Regression. New York: Wiley, 1989.

[33] Universal Mobile Telecommunications System (UMTS), Spacial Channel Model for Multiple Input Multiple Output (MIMO) Simulations, ETSI TR 125996 V6.1.0, Sep. 2003.

[34] Y. Ko and M.-S. Alouini, "Estimation of Nakagami-m fading channel parameters with application to optimized transmitter diversity systems," IEEE Trans. Wireless Commun., vol. 2, no. 2, pp. 250-259, Mar. 2003.
[35] C. Tepedelenlioglu and P. Gao, "Practical issues in the estimation of Nakagami-m parameter," in Proc. IEEE Globecom 2003, Dec., vol. 2, pp. 972-976.

[36] Y. Chen, N. C. Beaulieu, and C. Tellambura, "Novel Nakagami- $m$ parameter estimator for noisy channel samples," IEEE Commun. Lett., vol. 9, no. 5, pp. 417-419, May 2005.

[37] D. V. Djonin and V. K. Bhargava, "On the influence of the power profile on diversity combining schemes," IEEE Trans. Wireless Commun., vol. 3, no. 4, pp. 1854-1861, Sep. 2004.

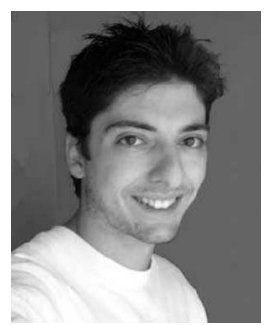

Athanasios S. Lioumpas (S'06) was born in Thessaloniki, Greece, in 1982. He received the Diploma in electrical and computer engineering in 2005 from the Department of Electrical and Computer Engineering, Aristotle University of Thessaloniki, Thessaloniki, where he is currently working toward the Ph.D. degree.

His current research interests include digital communications over fading channels, diversity techniques, and mobile radio communications.

Mr. Lioumpas is co-recipient of the Best Paper Award of the Wireless Communications Symposium (WCS) in IEEE International Conference on Communications (ICC' 07), Glasgow, U.K., June 2007.

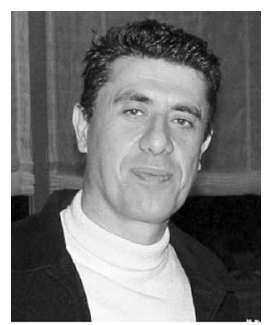

George K. Karagiannidis (M'97-SM'04) was born in Pithagorion, Samos Island, Greece. He received the University and Ph.D. degrees in electrical engineering from the University of Patras, Patras, Greece, in 1987 and 1999, respectively.

From 2000 to 2004, he was a Senior Researcher at the Institute for Space Applications and Remote Sensing, National Observatory of Athens, Greece. In June 2004, he joined Aristotle University of Thessaloniki, Thessaloniki, Greece, where he is currently an Assistant Professor in the Electrical and Computer Engineering Department. His current research interests include wireless communications theory, digital communications over fading channels, cooperative diversity systems, cognitive radio, satellite communications, and free-space optical communications. He is the author or coauthor of more than 70 technical papers published in scientific journals and presented at international conferences. He is also a coauthor of two chapters in books and a coauthor of the Greek edition of a book on mobile communications. He serves on the editorial board of the EURASIP Journal on Wireless Communications and Networking.

Dr. Karagiannidis has been a member of Technical Program Committees for several IEEE conferences. He is a member of the editorial boards of the IEEE TRANSACTIONS ON COMMUNICATIONS and the IEEE COMMUNICATIONS LETTERS. He is co-recipient of the Best Paper Award of the Wireless Communications Symposium (WCS) in IEEE International Conference on Communications (ICC'07), Glasgow, U.K., June 2007. 